\title{
Aplicación de un índice de estabilidad fisiológica en unidad de cuidados intensivos pediátrica
}

\author{
M.C. Enrique Paris M.' ; M.C. Mario Cerda S.' ; M.C. Pedro Lorca F. '; Javier Lagos R. ${ }^{3}$; \\ Carmen Otero $F^{3}{ }^{\text {; }}$ Liliana Ptina $P^{3}{ }^{3}$ : Estad. Luis Villarroel del $P_{1}^{4}$.

\section{Physiologic stability index and mortality in patients of a pediatric intensive care unit}

\begin{abstract}
This study was conducted to evaluate usefulness of the physiologic stability index (PSI) in a pediatric intensive care Unit (PICU) of a University based hospital at Santiago, Chile. Subjects include all patients ( $n=152)$ consecutively admited to our PICL between april 1987 and august 1988. The PSI system was applied during the first four days from admission. The age of the patients ranged from 10 days to 15 years. Scores obtained from survivors and non survivors were compared using an analysis of variance. For maximum PSI, survivors had significantly lower scores (9.39 points) that non survivors (21.71 points). Multuple logistic regression was used to display associations between mean obtained scores and probability of death and a curve was plotted which is sirnilar to that obtained in previous experiences from other countries.
\end{abstract}

(Key words: physiologic stability index, PSI, mortality, pediatric intensive care.)

El paciente crítico necesita y recibe una cantidad enorme de recursos para mantener sus posibilidades de sobrevivencia y para ayudar su progreso hacia una recuperación satisfactoria.

Se han llevado a cabo variados esfuerzos para evaluar el funcionamiento de las unidades de cuidados intensivos (UCI) pediátricas y se han desarrollado diferentes sistemas para clasificar los pacientes que ingresan a ellas, determinar sus necesidades y su pronóstico. Los sistemas de. satrollados para clasificar a los pacientes están destinados a calificar la severidad de la enfermedad, los requerimientos terapéuticos, la eficacia del tratamiento y el pronóstico ${ }^{t-3}$. Debido a la gran variedad de causas de hospitalización en las LCI pediátricas el sistema para calificar a los pacientes debe ser lo suficientemente versátil para ser aplicado a diferentes problemas y edades. Existen varios indices de calificación de gravedad de los pacientes, como

1. Unidad de Cuidado Intensivo Pediátrico, Servicio de Pediatría, Hospital Clínico Universidad Católica de Chile.

2. Médico Becario Pediatría, Universidad Católica de Chile.

3. Alumnos de la Carrera de Medicina de la Universidad Católica de Chile.

4. Estadístico de la Escuela de Medicina de la Universidad Católica de Chile. por ejemplo el Sistema de Clasificación Clínica (CCS) o la puntuación basada en Intervención Terapéutica (TISS), que miden en forma indirecta la severidad de la enfermedad en base a una evaluación de las necesidades terapéuticas. Knaus desarrolló, además, un sistema de clasificación basado en alteraciones fisiológicas (APACHE) aplicable a los adultos. Sin em. bargo, estos procedimientos de evaluación no existían para pediatría hasta el año 1984 en que se publicó la validación de un indice de estabilidad fisiológica para ser aplicado en niños (PSI $^{4}{ }^{4}{ }^{5}$. Este indice clasifica la severidad de la enfermedad a partir del grado de alteración de 7 grandes sistemas fisiológicos e incluye 34 variables adaptadas a pacientes menores de un año $y$ mayores de un año.

Los objetivos de este trabajo fueron ensayar la factibilidad de aplicar el PSI a los pacientes que ingresen a nuestra unidad de cuidados intensivos pediátricos, evaluar los criterios de ingreso a dicha unjdad en base a las calificaciones promedio de los pacientes ingresados y el funcionamiento de la misma correlacionando la mortalidad con las calificaciones asignadas.

\section{MATERIAL Y METODO}

Se aplicó el PSI a todos los pacientes $(n=152)$ que ingresaron a la unidad de cuidado intensivo del servi- 
cio de pediatría del Hospital Clínico de la Universidad Católica de Chile, entre abril de 1987 y agosto de 1988.

La UCI inició sus funciones en el mes de marzo de 1987 , cuenta con 6 camas y recibe pacientes médicos, quirúrgicos $\mathrm{y}$, sólo en forma excepcional, recién nacidos. Fi indice se aplicó durante 4 días consecutivos. Cada día se consignó el valor más alterado de cada una de las variables que se medía en un paciente determinado. El grado de anormalidad de cada variable fue calificado con 1,3 ó 5 puntos, dande una calificactón más alta no necesariamente implica una alteración mayor, cuantitativamente proporcional, de desviación sobre su valor normal, sino una variación de estabilidad que induce al médico a adoptaz una actitud de cambio en la terapia. Así se asignó 1 punto a aquellas alteraciones que no implican un cambio en la terapéuti$c a, 3$ puntos a las variaciones fisiológicas que inducen a cambios en la terapia y 5 puntos a las que ponen en peligro la vida del paciente, siendo, de este modo, más alto el valor de PSI cuanto mayor es la inestabilidad del paciente.

La edad de los pacientes fluctuó entre los diez días y 15 años. Las afecciones por las cuales ingresaron fueron médicas en su gran mayoria, encontrándose también politraumatizados, traumatismos encefalocraneanos y postoperados. De 152 pacientes, 79 eran del sexo masculino.

Una vez registradas las calificaciones diarias de cada paciente en los cuatro primeros días, ellas se sumaron día por día obteniendo así la puntuación total para cada uno. Siguiendo el esquema propuesto en el trabajo ya citado estas puntuaciones se dividiezon en las siguientes 7 categorías fijadas arbitrariamente:

$0-4,9 ; 5-9,9 ; 10-14,9 ; 15-19,9 ; 20-24,9 ; 25-29,9$; $>=30$.

Se compararon las calificaciones de los sobrevivientes y de los fallecidos mediante un sistema de análisis univariado. Aplicando un estudio de regresión logística se intentó determinar la asociación entre las calificaciones obtenidas y la probabilidad de muerte. Para el análisis de la significación estadística se utilizó la prueba $t$ de Student para muestras independientes. Además se compararon nuestros resultados con la cursa de probabilidad de mortalidad vs. calificación promedio obterida del trabajo ya citado ${ }^{4}$.

Se llamó calificación (puntuación) máxima a la suma máxima de puntos obtenida por un paciente o grupo de pacientes en cualquiera de los 4 días consecutivos. Se lamó calificación (puntuación) promedio al promedio de la suma de las calificaciones obtenidas a partir de los puntos de cada día, durante los días en que fueron reglstradas.

\section{RESULTADOS}

Se evaluaron 152 pacientes. La letalidad general fue de $9,5 \%$. La distribución de pacientes en relación a la calificación preestablecida fue la descríta en la tabla 1.

Al comparar las calificaciones promedio de los cuatro días medidos en sobrevivientes y fa-
Tabla 1

Distribución de pacientes según calificación

\begin{tabular}{lrcr}
\hline Pje. promedio & Vive & Fallece & Total \\
\cline { 2 - 4 } $0-4,9$ & 67 & 0 & 67 \\
$5-9,9$ & 43 & 1 & 44 \\
$10-14,9$ & 19 & 6 & 25 \\
$15-19,9$ & 7 & 2 & 9 \\
$20-24,9$ & 0 & 4 & 4 \\
$25-29,9$ & 2 & 0 & 2 \\
$>=30$ & 0 & 1 & 1 \\
Total & 138 & 14 & 152 \\
\hline
\end{tabular}

llecidos, mediante análisis univariado, el promedio en los sobrevivientes fue 6,60 puntos y en los fallecidos 16,94 puntos, siendo la diferencia estadisticamente significativa al aplicar la prueba $t$ de Student para muestras independientes (tabla 2).

Al comparar las calificaciones máximas de los sobrevivientes $y$ fallecidos, mediante análisis univariado, los primeros tuvieron 9,39 puntos $y$ los que murieron 21,71 puntos, siendo la diferencia estadísticamente significativa al aplicar la prueba $t$ de Student para muestras independientes (tabla 3).

Se realizó un análisis de regresión logística para correlacionar la probabilidad de muerte con la puntuación máxima, la puntuación pro-

Tabla 2

Calificaciones promedio

N Promedio D.E. Calif, mín. Calif. ınáx.

\begin{tabular}{lrrrrr}
\hline Fallece & 14 & $16,94 *$ & 7,68 & 7,75 & 35,00 \\
Vive & 138 & $6,60 *$ & 4,92 & 0,00 & 28,00 \\
\hline
\end{tabular}

$* p<0,001$

D.E. = Desviación estándar.

Tabla 3

Califīcaciones máximas

N Promedio D.E. Calif. min. Calif, máx.

\begin{tabular}{lrrrrr}
\hline Fallece & 14 & $21,71 *$ & 8,69 & 11,00 & 37,00 \\
Vive & 138 & $9,39 *$ & 6,60 & 0,00 & 34,00
\end{tabular}

${ }^{*} \mathrm{p}<0,001$.

D.E. $=$ Desviación estándar. 
medio y la edad de los pacientes. No se encontró correlación entre la edad de los pacientes y la probabilidad de morir. Se logró establecer una relación entre la probabilidad de muerte y la calificación promedio de los cuatro días, que permitió construir una curva de correlación para los pacientes hospitalizados en la UCI pediátrica del Hospital Clínico de la Universidad Católica, que revela que los pacientes con calificación de 25 puntos o más tienen sobre $50 \%$ de probabilidades de morir (figura 1 ).

Al comparar nuestra curva de probabilidad de fallecimiento vs. calificación promedio con la curva de Yeh-Pollack ${ }^{4}$, podemos advertir que ambas son similares, aunque para la misma suma de puntos la mortalidad en la curva original es levemente más alta que la nuestra (figura 2).

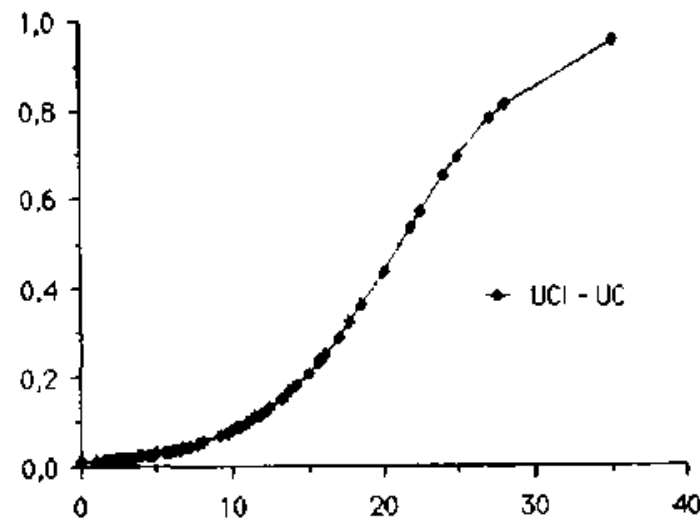

Figura 1: Probabilidad de muerte vs. Calificación promedio. UCI pediátrica U.C. Eje horizontal: Caljfücación promedio. Eje vertical: Probabilidad de muerte.

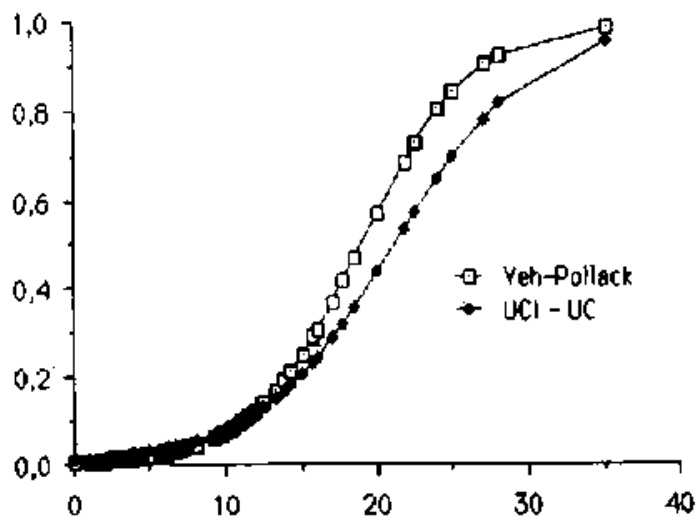

Figura 2: Probabiłidad de muerte vs. Calificación promedio. UCI pediátrica L.C. vs. Yeh-Pollack. Eje horizontal: Calificación promedio. Eje vertical: Probabilidad de muerte.

\section{DISCUSION}

Es posible aplicar a nuestra realidad el Indice de Estabilidad Fisiológica (PSI) que, mediante el registro de diferentes variables, permite obtener calificaciones máximas y promedios, que es posible correlacionar con la probabilidad que tienen nuestros pacientes de fallecer. Esto nos permite conocer los pacientes de riesgo y apoyarlos con todos los medios a nuestro al cance para lograr su recuperación.

Todos los pacientes con califícación promedio entre 0 y 4,9 sobreviven ( 67 pacientes), $41 \%$ del total estudiado, proporción que es atribujble, por una parte, a la disponibilidad de camas UCl $y$, por otra, indica que quizás debemos ser más estrictos en nuestros criterios de ingreso. Esto último también podria explicar que la letalidad en nuestra serie sea menor que en otras con mayores número de pacientes $y$ complejidad de problemas 4 .

Dos de los parámetros descritos permiten diferenciar los pacientes fallecidos de los vivos, entre los egresados de una UCI pediátrica, ellos son la calificación (puntuación) promedio y la calificación máxima que es posible asignarles con el método descrito.

El PSI, al abarcar tantas variables, es un método poco práctico. Es posible que mediante análisis estadístico se llegue a determinar con mayor precisión, con poblaciones mucho mayores que la nuestra ${ }^{6}$, cuales son las minimas variables que es necesario medir para obtener índices pronósticos, lo cual facilitaría la labor de registro e interpretación de los resultados obtenidos.

\section{RESUMEN}

Se aplicó el Indice de Estabilidad Fisiológica (PSI) a todos los pacientes ingresados a la UCI pediátrica del Hospital Clinico de la Universidad Católica de Chile en el período comprendido entre abril de 1987 y agosto de 1988 . La edad de los pacientes varió entre los 10 días de vida $y$ los 15 años de edad y se evaluaron 152 niños. El PSI fue aplicado durante los 4 primeros dias de estadia de dichos pacientes en la UCI y se obtuvo una calificación promedio que se correlacionó con la probabilidad de morir mediante un análisis de regresión logistica. Mediante un análisis de varianza se logró deterninar que existen diferencias significativas entre la califica- 
ción máxima de los no-sobrevivientes (21.71 puntos) y la calificación máxima de los sobrevivientes $(9,39$ puntos $)$, según la prueba $t$ de Student.

(Palabras clave: cuidados intensivos pediátricos, indice de estabilidad fisiológica, letalidad, pronóstico.)

\section{REFERENCIAS}

1. Cullen D.J.: Results and costs in intensive care. Anesthesjology, 1977; 47: 203-216.

2. Cullen D.J., Civetza J.M., Briggs B.A.: Therapeutic intervention scoring system: a metod for quan- titative comparison of patient care. Crit Care Med, $1974 ; 2: 57-60$.

3. Knaus W.A., Zimmermon J.E., Wagner D.P. Draper E.A., Lowrence D.E.: Apache: Acute physiology and chronic health evaluation: a physiologic based classification system. Crit Care Med, 1981; 9: 591-597.

4. Yeh T.S., Pollack M.M., Ruttimann U.E. et al.: Validation of a physiologic stability index for use in critically ill infants and children. Pediat kes $1984 ; 18: 445-451$

5. Pollack M.M. Yeh T.S., Ruttimann U.E., ef al.: Evaluation of pediatric intensive care. Crit Care Med 1984; $12: 376-383$

6. Pollack M.M., Getson P.R., Rustimann L.E. et al.: Efficiency of intensive care. A comparative analysis of eight pediatric intensive care units. JAMA $1987 ; 258$ : 1481-1486. 